\title{
36
}

\section{Equus: a QOS manager for distributed applications}

\author{
C.J. Sreenan and P.P. Mishra \\ ATET Bell Laboratories \\ 600 Mountain Avenue, Murray Hill, New Jersey, 07974, USA. \\ Telephone: +1-908-582-7685. Fax: +1-908-582-5192. \\ email: $\{$ cjs, partho\}@research.att.com
}

\begin{abstract}
This paper discusses QOS management for distributed multimedia applications, focusing on end-system support. We propose a new software framework for managing resources which is composed of two key elements: the QOS management and application design models. The former has at its core a QOS manager which is responsible for admission control, QOS translation and performance monitoring. It also provides mechanisms and policies for employing dynamic adaptation to manage application access to resources. The application model is used to simplify the problems of QOS specification and resource allocation/accounting. Preliminary experiences with a prototype of this system in a workstation environment are reported. This has been implemented as the Equus QOS management platform currently running over Unix System V.4.
\end{abstract}

Multimedia, quality of service

\section{Keywords}

\section{INTRODUCTION}

The combination of vastly increased transmission bandwidth along with the availability of sophisticated media compression algorithms has made possible a new breed of distributed multimedia applications. Efficiently supporting such applications involves redesigning systems components that were originally designed to support a less general set of applications. One of the key design issues is that of resource control: how should system resources be shared so as to satisfy the quality of service (QOS) requirements of applications while making efficient use of them.

The problem of QOS support for multimedia applications has been primarily addressed in the domain of computer networks. This is achieved by managing network resources, such as switch capacity, so that the stated QOS requirements of applications are met. Typical QOS parameters describe throughput, delay, jitter and loss rates. Using this information, Ferrari (1990) has shown how to negotiate for network resources between the source and 
sink of data streams. While much of this work is related to ATM, there are also efforts to accommodate QOS in the Internet, see Mitzel (1994).

Only recently has attention been drawn to the distributed nature of this problem and the need to manage end system resources, in addition to those in the network. Lack of QOS management for these resources can cause the benefits of a network QOS guarantee to be seriously diminished by the time the data actually reaches the output device. These end systems include presentation points such as PCs, home terminals and workstations, as well as servers for storage and multipoint bridging etc. In early systems this problem was avoided by using source and sink end points which were effectively dedicated to handling multimedia (often backed by custom hardware). This approach is expensive and unsuitable for applications which must share resources with other activities, e.g. executing on a workstation or making requests to a shared server. We have been exploring the role of QOS in this wider context, in terms of a software framework that is appropriate for end to end resource management.

In this paper we describe our architectural approach and discuss our experiences with a prototype implementation in an environment consisting of multiple workstations connected via Ethernet. Key to our proposal is that an application must specify its QOS requirements in a system independent way, relying on a QOS manager to deal with the specifics of different underlying resource schedulers. We also strongly advocate the use of a QOS interface which allows an application to state how it can adapt to different quality levels. Coupled with these features is an application model in which we adopt a modular approach to stream manipulation, simplifying the tasks of QOS specification, resource allocation and accounting. The prototype version of our Equus platform implements these proposals on Unix System V.4. A software H.261 video encoder and decoder demonstrate our application model in use with a QOS manager which translates specifications, controls admission and manages adaptation. The paper is organized as follows. Sections 2 \& 3 present our QOS and application design models respectively. Section 4 describes the prototype, and our initial results appear in Section 5. Section 6 concludes with a discussion of the results, related work and future directions.

\section{QOS MANAGEMENT MODEL}

Providing end to end QOS guarantees to applications demands a suitable architecture and policies for resource management. A QOS architecture has a set of basic functions: specification/negotiation, admission control, policing and scheduling. Application specifications describe traffic characteristics and performance requirements, which are translated in terms of resource demands and used in combination with knowledge of already existing applications to perform an admission control test. A policing mechanism aims to ensure that an application does not violate the stated traffic limits while the scheduling mechanism operates to try and meet performance requirements. In order to make use of QOS specifications in guiding system resource management we have identified a number of components, illustrated in Figure 1.

There are three basic components in this model: resources, applications and QOS managers. Examples of resources are CPUs, switches, links etc. Associated with a resource is a resource manager which enforces how a resource is shared by multiple users. We assume that groups of resources are located within certain resource domains. A resource 


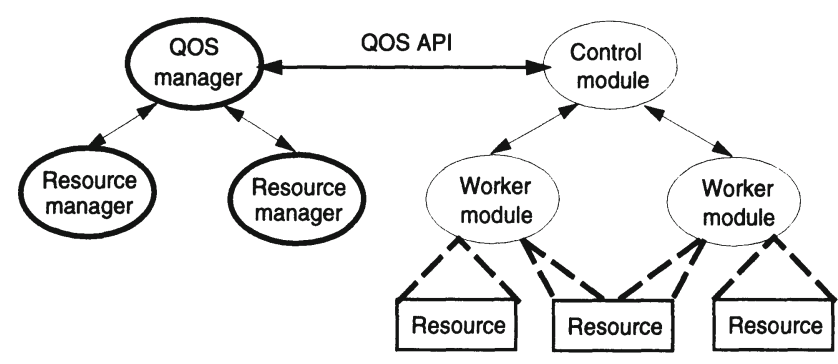

Figure 1 Software elements.

domain is an administrative boundary, access to which may have cost and/or security implications. An example of a domain is the set of resources comprising a workstation. Each application is assumed to be composed from a set of worker modules which handle streams, and whose operation is overseen by a separate control module. A stream is a single flow of continuous media $(\mathrm{CM})$ data originating from a source and following a path in which it is processed in sequence by an array of worker modules.

A QOS manager is an entity associated with each resource domain that is responsible for ensuring that the QOS requirements of applications are met when they only use resources that belong to QOS manager domain. The module based approach simplifies the task of specifying application performance requirements and allocating resources in a distributed system. There are two levels of specifications. First, worker modules have certain usage/demand patterns for the resources they use. Since access to the resource is scheduled by a resource manager, it is necessary to use the demands of a module and determine if there is a feasible schedule at each individual resource which satisfies the requirements without upsetting those of already admitted modules. In addition, the scheduling algorithms used by resource managers may vary, so the QOS manager has to map between the application specified requirements and the capabilities of the scheduler. At a higher level, the QOS across multiple different resources has to be negotiated: this task is also performed by the QOS manager in response to a request from the control module of an application. Each QOS manager operates within a defined resource domain, interacting with underlying resource managers to perform admission control. It also communicates laterally with other QOS managers in situations where a module requires the use of resources which are external to its domain. Our underlying philosophy here is that an application must specify its performance requirements in a manner which is system independent. It is the job of a QOS manager to map these requirements in terms of the type of resources and their availability status.

Our system is designed with the assumption that it is hard to precisely characterize the demand patterns or guarantee the requirements of an application. This is because of two reasons. First, traffic characteristics for a stream are hard to describe accurately. This is particularly the case for data which is compressed to produce a variable bit rate. Second, admission control depends on knowledge of the underlying resource capacities and how they are scheduled; this information is also difficult to obtain (and translate to) accurately. For these reasons, we advocate a more flexible approach, in which the ability of applications to perform limited adaptation based on resource availability is assumed. The 

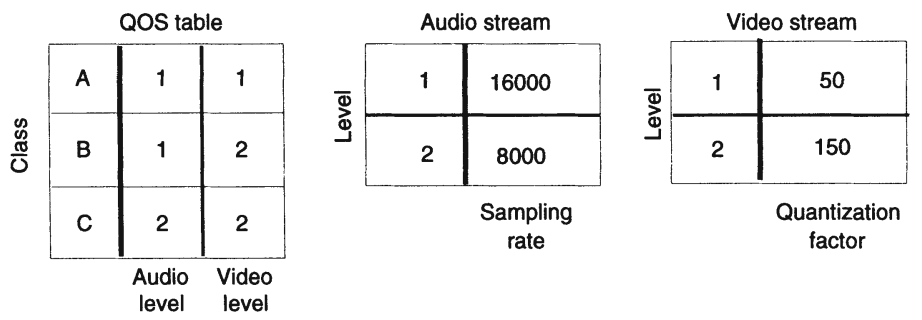

Figure 2 Example QOS specification.

type of adaptation referred to is where an application might relax its delay requirements or reduce its bit rate, e.g. by using a lower frame rate. Our system supports adaptation as a fundamental part of the QOS specification, and uses it in combination with performance monitoring mechanisms to renegotiate on behalf of applications when necessary.

This is achieved by introducing QOS levels that are used by an application to specify a scale of how its service can be degraded. This feature is also used during initial negotiations to deal with situations where sufficient resources are not available, or if it is desirable for the application to reduce usage charges by opting for a lower level of service quality. Inter stream relationships can easily be defined by having several QOS classes, and for each stream listing the QOS level it should operate at in that class. An example of such a specification is shown in Figure 2. Here is an application with an audio and video stream, each of which can operate at one of two possible QOS levels. A QOS table defines the relationship between the streams, indicating that both streams can be degraded, but video should be degraded in preference to audio. For simplicity this example omits details of other modules and end to end parameters. When used in combination with a relaxed admission control policy, this generalized model of adaptation should allow QOS control without excessive under utilization of resources and in settings such as workstations, which are less deterministic than might be desired. Note that adaptation decisions in our model are seen as taking place at fairly coarse grained time scales, say of the order of several seconds.

There are several benefits of the QOS manager centric approach we advocate in this paper, in which a QOS manager acts as an agent for the application. It provides a uniform interface to system resource management, isolating applications from the existence of heterogeneous schedulers. This is in keeping with the principle of QOS being a declaration of what an application wants, rather than how it is to be provided. Using a QOS manager relieves the application from the potentially complex task of negotiating over multiple resources. It also allows the process of negotiation to be optimized by maintaining state information on resource usage which can be used as hints, for example in selecting a target location at which to execute a module. We next describe our application model and its interaction with the QOS manager in greater detail. 


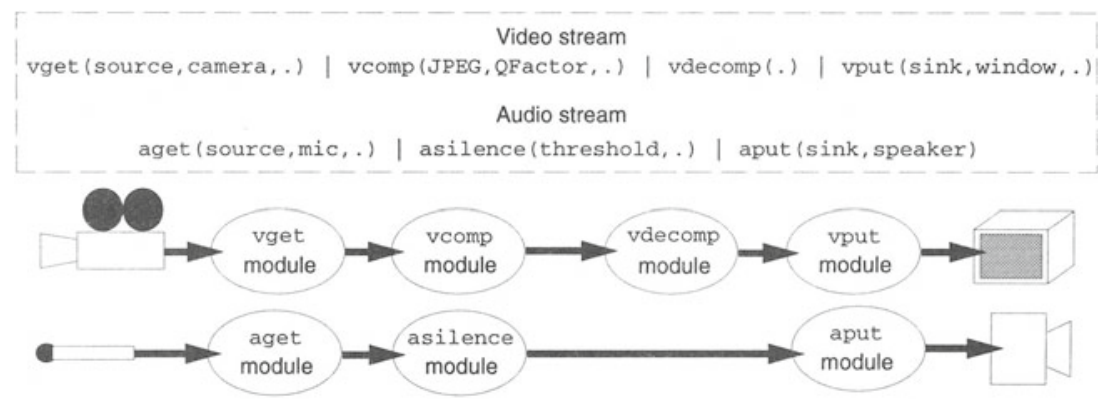

Figure 3 Example of an application using SPFs.

\section{APPLICATION MODEL}

We propose a model for distributed multimedia application and server design which simplifies QOS specification and resource management. This model is based on the principles of functional decomposition and separating control from data, and differs from the conventional view of a multimedia application as a monolithic process. As previously defined, in this model an application is composed from a set of worker modules which handle streams, and whose operation is overseen by a separate control module. A stream is a single flow of CM data originating from a source and following along a path in which it is processed in sequence by an array of worker modules. Each worker module is a thread of execution which implements a stream processing function (SPF). Basic SPFs are those which capture CM from input devices such as cameras and microphones. More sophisticated SPFs perform operations based on the CM content, examples being compression and silence detection. Predefined worker modules for common stream processing functions may be reused through a library, so that writing a multimedia application becomes as trivial as providing a script which for each stream identifies sources, sinks and the desired SPFs and their parameters. This would then be used to instantiate modules and the segments which form the stream path. An example is given in Figure 3.

Although models of this form have traditionally been proposed for reasons of correctness or reuse, our primary motivation for proposing this model is to facilitate QOS based resource management. The modular approach provides several benefits in this context. Since each module is designed to perform a single SPF, it is easier to characterize its behavior when compared with that of describing the application as a whole. In addition, by associating performance requirements with each module we introduce a layer of indirection which facilitates mapping performance specifications to heterogeneous resources and schedulers. Each module has performance information which defines its characteristics in terms of resource demands for a given set of input parameters, e.g. image size, frames per second. For common operations, this relieves the application programmer of having to provide the SPF and derive a description of its behavior. The decomposition of an application into simpler worker modules also makes it possible to better characterize the transformation of a stream as it passes through a module. Moreover, this model makes it feasible to distribute some of the SPFs onto different physical resources, depending 
on resource availability. Finally, a module is the entity to which system resources are allocated making it feasible to do resource accounting on a per module basis, simplifying the situation where modules execute on several remote systems on behalf of a single application.

As a related point, servers should also be designed in a modular fashion to allow finer control over resource allocation and accounting. A server distinguishes between the modules and the resources they require which it operates on behalf of different clients. Unlike the more conventional approach, for example with the $\mathrm{X}$ server, this approach allows servers to differentiate between the service provided to each client. The need for such a structure becomes evident when one wishes to avoid situations where for example the RPCs of one client can have a detrimental effect on the performance received by other clients. This has parallels with the effect of QOS crosstalk as discussed by Tennenhouse (1989) in relation to the design of layered protocol stacks.

Of course the difficult task of how one could characterize a new SPF remains. In order to determine the resource requirements for a module to execute an SPF we need a SPF transform which can determine a list of resource requirements and output traffic characteristics, given the input traffic characteristics, functional/quality control parameters and a description of target resource types. An application's QOS specification for a stream then becomes a list of SPFs and their parameters, along with end to end performance bounds on delay, jitter and losses. It may also be desirable to allow higher level relationships to be explicitly defined, e.g. inter stream and application, although the benefits must be weighed against the additional complexity of negotiating for resources. For the purpose of deriving a stream transform a simulation environment seems appropriate. This would allow the SPF developer to build up a performance and resource usage profile in a range of different situations. By its nature, this information can only be used to predict the expected resource requirements of an associated module. To increase the accuracy, one could augment a transform with historical information gathered and reevaluated each time the $\mathrm{SPF}$ is executed. Maintaining this information in terms of a set of particular contexts, e.g. the invoking application type and/or user, provides additional input. An example for a conferencing application is in estimating the expected amount of scene movement coming from a given source by relying on the recorded mannerisms of the remote user in question.

\section{PROTOTYPE SYSTEM}

We have designed and built a prototype to evaluate the proposed software framework. The system exists as a distributed software platform called Equus, which provides an environment for QOS management over a cluster of workstations. We provide the core facilities for QOS based resource management at the end system, allowing experimentation with policies for specifying QOS requirements, and performing admission control and adaptation. In the current prototype we focus on a single resource, namely CPU access. The CPU resource manager in the prototype is that of UNIX System V.4, which allows fixed priorities to be assigned to processes placed in the realtime class. This allows us more control than conventional UNIX time sharing schedulers, but is far from the newer type of contract driven systems proposed for CM by Hyden (1994) and Mercer (1994). The use of the System V.4 realtime class to support a mix of CM and other applications without employing adaptation has been shown by Nieh (1993) to lead to potentially severe 
performance problems. Unlike that work, one of our key goals was to experiment with an application's ability to operate at, or adapt to, different levels of quality when dealing with situations where the desired resources are unavailable. The environment we have chosen is more appropriate for testing adaptation based policies due to the less deterministic nature of the guarantees provided by the scheduler. The next section gives details of the application SPFs that have been ported for use within the Equus platform. It is complemented by Section 4.2 which describes the current version of the QOS manager.

\subsection{Application SPFs}

A set of SPFs were selected and ported so that they could be made available to applications using the Equus platform. The current suite of applications come from the public domain IVS system described by Turletti (1993), which provides audio/video conferencing over the Internet, in addition to storage and retrieval of CM files. Video is compressed using the H.261 standard, producing a variable bit rate stream. The current software testbed is illustrated in Figure 4, which shows UNIX processes executing modules for application control and the four SPFs which are available, namely:

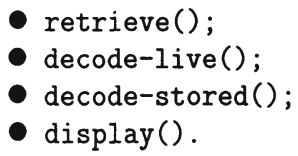

There are a couple of points to note. There are two types of applications used: a live simplex video connection for ivs-conf; and ivs-replay which retrieves and presents a locally stored video file. The host being used for presentation is the QOS domain to be managed, rather than the host used for capture. Because each stream uses a display $\mathrm{SPF}$, one would assume that it would be instantiated as several modules, each serving a single stream. Instead, there is only a single such module used by several streams. This is an undesirable consequence of using the installed $\mathrm{X}$ window system server to present video, and could be avoided by redesigning the server along the lines suggested in Section 3, to recognize the different performance requirements of its clients. An application control module is required to register with the QOS manager prior to commencing stream processing, in order to allow admission control. Its QOS specification consists of a list of the SPFs involved, and information about their expected resource requirements when operating at each stated QOS level. The number of such levels and how they relate to adaptation is at the discretion of each application. Each application control module also provides a callback interface which is used by the QOS manager to request that it adapt, by changing the level at which its worker modules operate.

The video encoder as supplied allows either manual sender control over maximum frame rate and/or bandwidth values, or can be asked to automatically adjust its output in response to network congestion (detected at the sink decoder). For the experiments, the encoder was set to manual control with a maximum bandwidth of $100 \mathrm{Kbps}$ (enforced by dynamically changing quantization and movement threshold limits), and modified to allow the maximum frame rate to be set explicitly by the remote receiver, which is used by ivs-conf when changing QOS levels. For the ivs-replay application a similar mechanism was installed to alter the frame rate of the retrieve SPF. In reality, changing the frame 


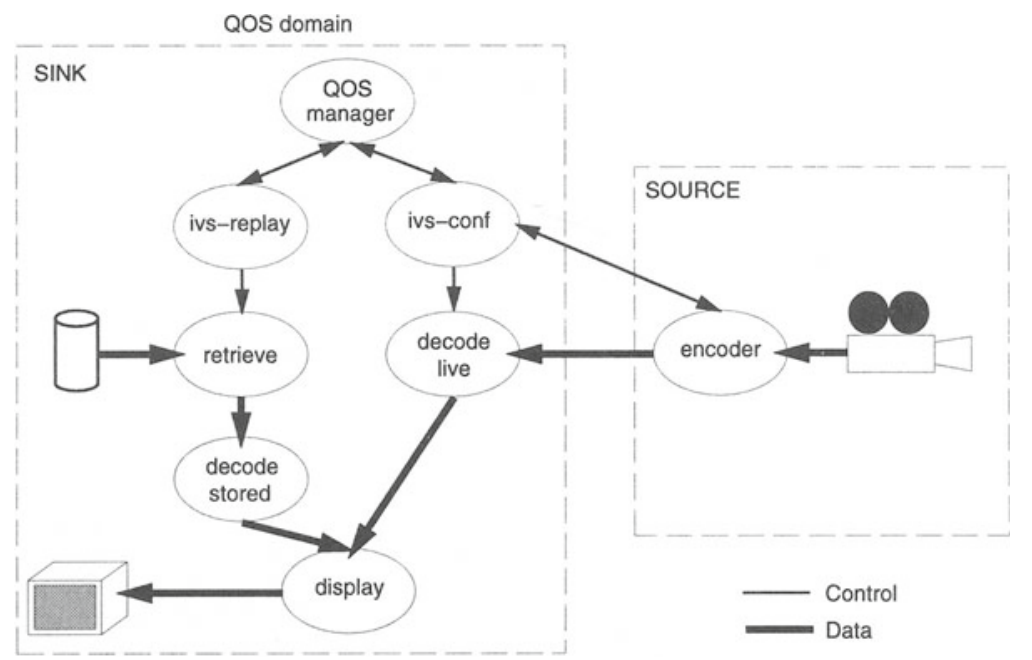

Figure 4 Software testbed for the prototype.

rate of stored video is undesirable, so a mechanism which can either discard frames or parts thereof (perhaps using a layered encoding) would be more suitable. This comment also has some value for live streams, where in multipoint situations it is highly undesirable to reduce the rate of an encoder to the lowest level demanded by any one receiver.

Each application must provide details of the expected behavior of its SPFs. For common SPFs this information is likely to be advertised, perhaps along with the SPF definition. In the present system, each of the four SPFs were profiled when operating at approximately 5,10 and 15 frames per second and for each of three different types of stream. The type of a stream in this case is defined by the average degree of movement which is expected, producing:

- low: relatively motionless view, e.g. from a surveillance camera;

- mod: moderate movement view, e.g. talking-head in a conference;

- high: potentially significant motion view, e.g. TV coverage.

The amount of data produced (and computation required) by H.261, like many compression algorithms, is highly dependent of the degree of movement between frames. Thus, it is important that the control module be aware of the expected stream type when selecting which set of data to send to the QOS manager. For ivs-replay this quality is stored at the time the stream is recorded, while the ivs-conf user states it as a command line argument. One could invent more elaborate methods, such as using the name of the stream source as an index to a table of behavior during previous conferences. In reality a stream is likely to diverge from its expected type, in which case an application, when asked to adapt, may wish to consider renegotiating with the QOS manager rather than reducing the QOS level. 
Table 1 SPF details for each stream type/QOS level (in msecs per second)

\begin{tabular}{cccccccccc}
\hline SPF & \multicolumn{3}{c}{ low } & \multicolumn{4}{c}{ mod } & \multicolumn{3}{c}{ high } \\
\cline { 2 - 11 } & 0 & 1 & 2 & 0 & 1 & 2 & 0 & 1 & 2 \\
\hline retrieve & 3 & 5 & 8 & 4 & 7 & 10 & 4 & 7 & 11 \\
\hline decode-live & 20 & 40 & 60 & 55 & 100 & 135 & 90 & 130 & 165 \\
\hline decode-stored & 20 & 40 & 60 & 45 & 90 & 135 & 55 & 110 & 165 \\
\hline display & 42 & 83 & 125 & 42 & 83 & 125 & 42 & 83 & 125 \\
\hline
\end{tabular}

The value provided in the prototype for each SPF/level pair is a measure of expected average CPU utilization over a one second period. Note also that the IVS encoder is controlled using a maximum frame rate value, so that its actual frame rate can sometimes be one or two frames per second less than predicted. A more sophisticated interface which also combines details of peak utilizations and burst probabilities is desirable for a furure version. Table 1 shows the values for each SPF as used in the experiments described in Section 5. There are a couple of points to note. The retrieve SPF has a very low utilization as it simply transfers data from disk to the decoder. The utilization of the display SPF depends on the frame rate (and of course the image size which is fixed at grey $176 \times 144$, known as Quarter CIF) but not by the type of stream. Stream type is important however, for the decode SPFs as can be clearly seen. An interesting point is why a distinction is drawn between decode-live and decode-stored which perform the same function. For the former, changing the frame rate results in adjusting the encoder, which has a side effect of increasing the per frame CPU utilization. Hence, the per frame CPU utilization at $15 \mathrm{fps}$ is less than that at $10 \mathrm{fps}$ (and similarly for $5 \mathrm{fps}$ ). This is because of increasing the delay between frame capture, which allows for more movement and hence a larger amount of data is generated with respect to the previous frame. This is not the case for decode-stored where the video is recorded at $15 \mathrm{fps}$ and, as described previously, is simply retrieved as a lower rate as needed.

\subsection{QOS manager}

The QOS manager accepts QOS specifications from applications and provides mechanisms and policies for admission control, performance monitoring and adaptation. It runs as a UNIX process, and in its current version, recognizes a single resource manager (for the CPU). An application interacts with the QOS manager by linking to a library which has the following form:

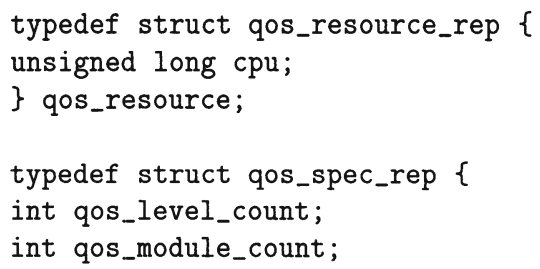




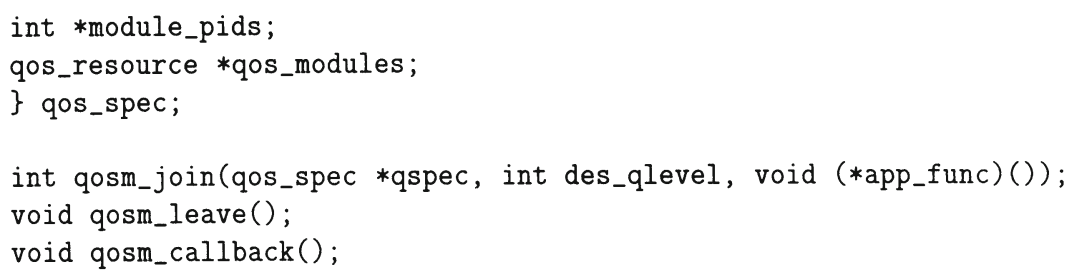

Processes are forked, each acting as a module executing a given SPF, and wait for the parent (control module) to try and obtain admission clearance from the QOS manager, at which point they are instructed to continue or terminate. Based on the stream type, the application selects utilization values for each module as described in the previous section and passes them as part of the QOS specification. Other elements are the process identifiers of each module and the desired QOS level. The control module invokes the callback function in response to data arriving on the communications endpoint created by the library for receiving adaptation requests from the QOS manager. This, in turn, invokes the application defined function which leads to a change in the current QOS level. At present the QOS manager accepts only two types of application requests: negotiate or withdraw.

Influenced by the need to minimize the effects of the shared display module, CPU scheduling priorities are assigned in decreasing order of importance as follows:

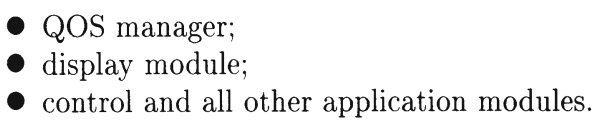

Admission control is a matter of comparing the total resource requirements of a new request against the current allocation in terms of milliseconds of CPU time per second. The latter is initialized so that the admission control algorithm should never allow utilization to exceed a preset value. Should there be insufficient resources to admit at once, the QOS manager invokes the adaptation algorithm with the aim of reducing the QOS levels of existing clients.

The QOS manager can operate with different adaptation policies, each defined by how it assigns and uses client priorities (note that these are not to be confused with CPU scheduler priorities). The present policy assigns priorities to clients such that more recent clients have a higher priority. When adapting downwards the client with the lowest priority is considered first (and vice versa when a client goes away and its resources become available). Thus, for example, an incoming request from a newly created video conferencing client can result in already running clients to be asked to reduce their QOS level. This policy has also been recommended by Jones (1993) based on practical experiences in dealing with resource contention between CM streams. There are many alternatives, including schemes which consider resource needs or application priorities assigned statically by the workstation user (perhaps in the style of a .qosinitrc file). The QOS manager monitors actual module utilization by examining their entries in \proc. This is performed regularly (currently every 10 seconds) and if a client is exceeding its stated limit, results in a request that it adapt down to a lower level if such exists. 


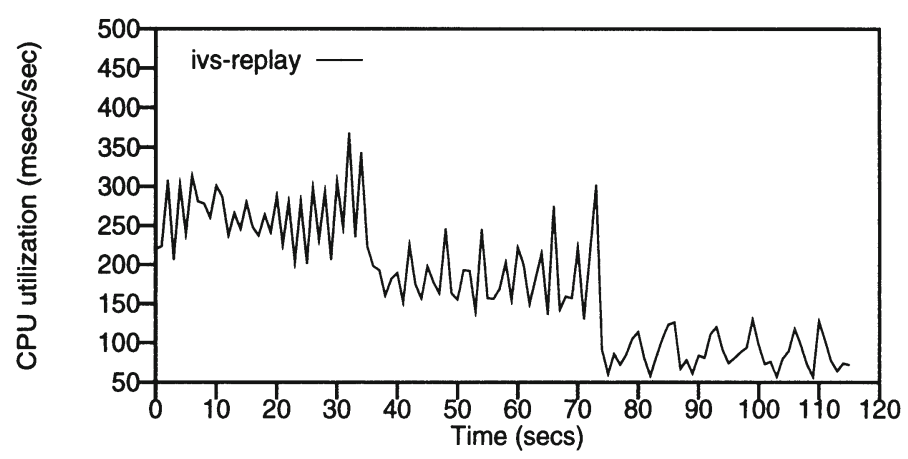

Figure 5 Adaptation mechanism in operation.

\section{EXPERIMENTAL RESULTS}

The Equus prototype has been used to experiment with workstation QOS management. This section contains preliminary results obtained using Equus with multiple applications over Solaris 2.3 on a SUN SPARCstation-10. Video is obtained either locally from a disk, or across a lightly loaded Ethernet from another SPARCstation-10 with a Parallax board.

Figure 5 illustrates the basic adaptation mechanism in operation. Here we have a single ivs-replay client, with the relationship between QOS level and CPU utilization clearly discernible. This is complemented by Figure 6 which demonstrates the current adaptation policy when running two instances of ivs-replay and one ivs-conf, with the maximum utilization set to $75 \%$. This graph plots CPU utilization over time for the clients, by simply using the sum of the utilization of the constituent modules. Initially the ivs-replay modules are executing a high movement stream at level 2, their highest quality level. The arrival of ivs-conf results in an admission request to the QOS manager, which asks the oldest client to adapt downwards by two levels in order to admit the new client. Later on when the ivs-conf terminates, that same client is asked to adapt upwards. This is in keeping with the current policy because the younger client is already operating at its desired quality level. The tail off at the end of ivs-conf is caused by the conference termination.

The next pair of experiments examine adaptation in comparison with the more conventional scenario where resource contention results in interference. Four ivs-replay clients are used, each playing back a high movement stream, with three commencing at the beginning of the experiment, and the other introduced half way through. Figure 7 shows the result in the conventional situation where a QOS manager is not present. It can be seen that the fourth client results in overload, interfering with the existing clients and resulting in increased average frame periods and variance. Naturally, this effect would be even more noticeable at higher loading. Figure 8 illustrates the case where we use a QOS manager to control admittance. The maximum utilization is set to $100 \%$ in this case for a fair comparison. Here the oldest two clients are adapted down by two levels each in order to admit the new client and avoid uncontrolled interference. Notice that the detrimental effect on frame period is lower for each stream in comparison to the previous case. 


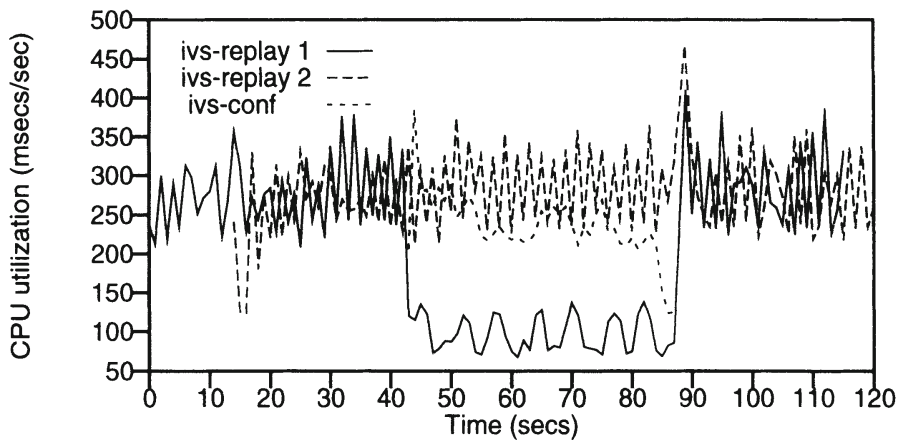

Figure 6 Adaptation policy in operation.

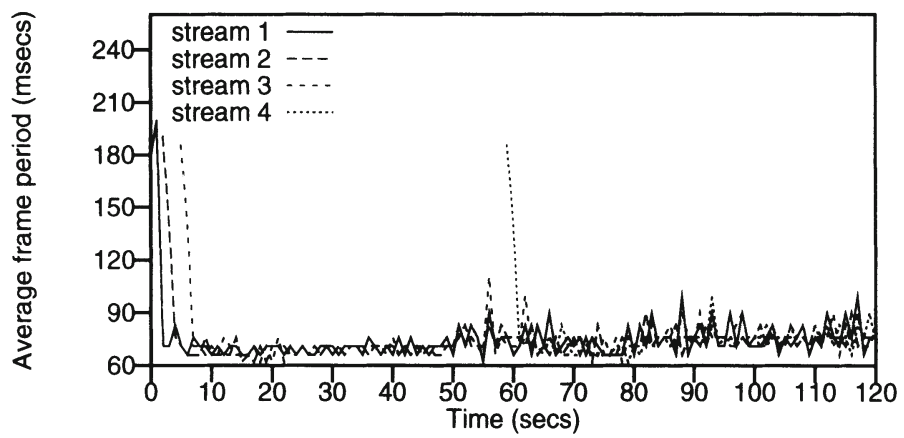

Figure 7 Overload scenario in a conventional workstation.

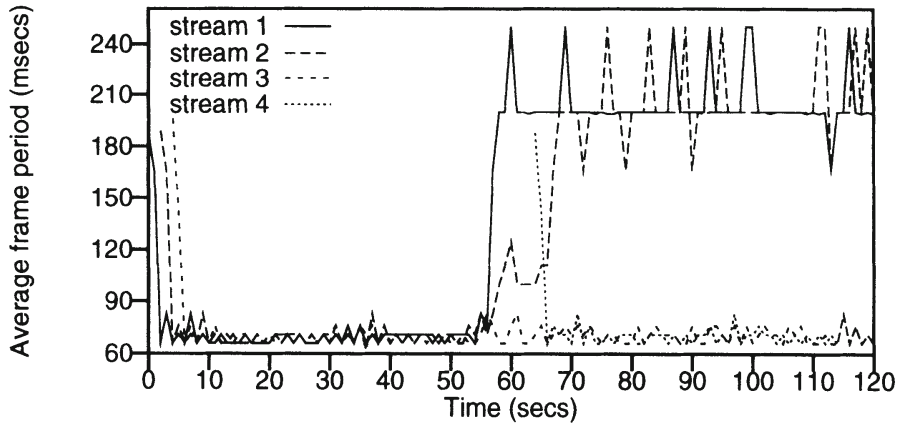

Figure 8 Overload scenario when using a QOS manager. 


\section{DISCUSSION}

We have presented and evaluated new system and application models for QOS management. At the core of the system is a QOS manager which accepts application specifications describing QOS requirements. The form of specification derives from our application model, and consists of end to end parameters and a list of SPFs for each stream. We argue that this modular approach simplifies specification, characterizing resource/performance requirements, and resource allocation/accounting. Key to our philosophy is that QOS specifications must isolate the application from details of the underlying resource schedulers. To this end, the QOS manager performs translation and manages adaptation based on specified QOS levels for each stream, and its relationship with other streams in the application.

Our focus with the current version of the Equus prototype has been on CPU access in the workstation environment. Several SPFs were ported to operate within the QOS platform, allowing us to experiment with both live and stored video applications. When initiated, these applications enter a negotiation phase with the QOS manager which determines admission. If admitted, an application can be asked to adapt its current service quality (and hence resource requirements) based on decisions made by the QOS manager. Our evaluation consisted of a set of preliminary experiments which demonstrated the core functionality of the platform, including admission control, monitoring and adaptation. A direct comparison between a conventional environment and a QOS managed one was also presented, illustrating the higher predictability of the latter. Regarding experimentation, our initial aim has been to evaluate adaptation for supporting $\mathrm{CM}$ in a workstation environment. With the mechanisms in place, this allows us to explore other policies for admission control and adaptation, using more detailed QOS specifications. Future work includes extensions for multiple and distributed resource managers.

Related work on QOS support for distributed applications can be broadly divided into two categories. Architectural proposals such as QOS-A by Campbell (1992) and IMAC by Nicolaou (1990) consider extending transport level QOS abstractions up to the application level. At a lower level, work on CPU scheduling for multimedia has been investigated by Anderson (1990), Hyden (1994) and Mercer (1994). Equus differs from these projects in two main aspects. Firstly, our proposal addresses the issue of specifying QOS at a high level for (potentially complex) distributed multimedia applications using multiple resources. This task is considerably simplified by our application model and the use of SPFs. Secondly, we assume heterogeneous resource schedulers running in environments which may be less deterministic than desired. We isolate applications from this substrate using a QOS manager to controls adaptation based on application specified QOS levels. Independently to our work, Tennenhouse (1994) has developed a modular approach to building multimedia applications which also incorporates a notion of graceful degradation.

\section{REFERENCES}

Anderson, D.P., Tzou, S., Wahbe, R., Govidan, R. and Andrews, M. (1990) Support for continuous media in the DASH system. Proceedings of the 10th International Conference on Distributed Computing Systems, 54-61.

Campbell, A., Coulson, G., Garcia, F. and Hutchison, D. (1992) A continuous media 
transport and orchestration service. Proceedings of the ACM SIGCOMM '92 Conference, Maryland, USA, 99-110.

Ferrari, D. and Verma, D.C. (1990) A scheme for real-time channel establishment in widearea networks. IEEE Journal on Selected Areas in Communications, 8(3), 368-79.

Hyden, E.A. (1994) Operating system support for quality of service. Computer Laboratory, University of Cambridge, Cambridge, England, Ph.D. thesis and technical report 340.

Jones, A. and Hopper, A. (1993) Handling audio and video streams in a distributed environment. Proceedings of 14 th ACM Symposium on Operating Systems Principles.

Mercer, C.W., Savage, S. and Tokuda, H. (1994) Processor capacity reserves: operating system support for multimedia applications. Proceedings of the International Conference on Multimedia Computing and Systems, 90-9.

Mitzel, D.J., Estrin, D., Shenker, S. and Zhang, L. (1994) An architectural comparison of ST-II and RSVP. Proceedings of the IEEE INFOCOM '94 Conference, 716-25.

Nicolaou, C. A. (1990) A distributed architecture for multimedia communication systems. Computer Laboratory, University of Cambridge, Cambridge, England, Ph.D. thesis and technical report 220 .

Nieh, J., Hanko, J.G., Northcutt, J.D. and Wall, G.A. (1993) SVR4 UNIX scheduler unacceptable for multimedia applications. Proceedings of the 4 th International Workshop on Network and Operating System Support for Digital Audio and Video, Lancaster, England, 35-47.

Tennenhouse, D.L. (1989) Layered multiplexing considered harmful. Proceedings of the IFIP Workshop on Protocol Design for High-Speed Networks, Zurich, Switzerland.

Tennenhouse, D.L., Adam, J.F., Carver, D., Houh, H.H., Ismert, M., Lindblad, C.J., Stasior, W., Wetherall, D., Bacher, D., and Chang T. (1994) A software-oriented approach to the design of media processing environments. Proceedings of the International Conference on Multimedia Computing and Systems, 435-44.

Turletti, T. (1993) H.261 software CODEC for videoconferencing over the Internet. INRIA, Sophia-Antipolis, France, Technical report 1834.

\section{BIOGRAPHY}

Cormac J. Sreenan is a Member of Technical Staff in the Networked Computing Research Department at AT\&T Bell Laboratories, Murray Hill, New Jersey, USA. He received a Ph.D. in Computer Science at the University of Cambridge in 1993 for work on continuous media synchronization. Prior to that he received B.Sc. and M.Sc. degrees in Computer Science at University College Cork, Ireland. His research interests include operating systems, networked multimedia and distributed systems.

Partho Mishra received the B.Tech. degree from the Indian Institute of Technology, Kharagpur, in 1988, and the M.S. and Ph.D. degrees from the University of Maryland, in 1991 and 1993, all in Computer Science. He is currently a Member of Technical Staff in the Networked Computing Research Department at AT\&T Bell Laboratories in Murray Hill, New Jersey, USA. His research interests are in the design and analysis of integrated service networks. 